\title{
La Missione Evangelica e l'Indipendenza Albanese
}

\section{All' Inizio del XIX Secolo le Società Evangeliche Allaciarono i Primi Contatti con Gli Intelletuali Albanesi}

L'Albania del dicianovesimo secolo fu coinvolta in piena dalle idee della dottrina evangelica. Terra di un popolo che apparteneva alle due confessioni cristiane ( cattolica e ortodossa) e quella mussulmana, fu fertile anche per le idee del protestantesimo. I centri di radiazione furono La Società Biblica Britannica e Straniera nato nel 1804 e II Bordo Americano attivo dal 1810. II punto di riferimento per le due società fu la "Casa Biblica" eretta nel 1872 a Costandinopoli. (Quanroud, 1998, p. 33) E proprio da lì partirono i missionari evangelici, che attraversando la famosa "Via Egnatia" arrivarono al cuore dei territori degli albanesi. Una parte dell' èlite albanese accolse con fervore le nuove idee, trovando in loro un nuovo modo di impostarsi ai problemi politici, economici e culturali che imperversavano l'Albania divisa in vilaieti e sotto il dominio ottomano. II primo contatto tra La Società Biblica Britannica e Straniera e la popolazione albanese avvenne quando lo scozzese Rev. Robert Pinkerton cominciò ad interessarsi della traduzione dei Vangeli in albanese. (Young, 2011, p. 22) I| 28 agosto 1816 || Rev. R. Pinkerton scrisse alla S.B.B.S. : "La Società Biblica Brittanica e Straniera deve prestare tanta attenzione all' approvvigionamento degli albanesi con almeno II Nuovo Testamento [ compresi I Vangeli] nella loro lingua... ovviamente l'approvvigionamento degli albanesi col Nuovo Testamento nella loro lingua sarebbe uno scopo degno delle sue previe e zelanti fatiche" (Clark, 2012, p. 11). Tra gli argomenti presentati da lui spiccava quello secondo cui; " la nazione albanese occupava una buona parte dell' lliria antica e dell' Epiro, parlava una particolare lingua diversa da quella slava, greca,turca e latina e che per quelli albanesi che erano cristiani ortodossi, la liturgia si espletava in lingua greca, una lingua incompressibile per il popolo e per una parte del clero". (Clayer, 2012, p. 163) . Dopo l' incontro organizzato a Costandinopoli tra Rev. Pinkerton e Vangjel Meksi si aprì la strada alle traduzioni in albanese .

Alexander Meksi (1770-1823) compì gli studi a Janina ed ebbe un esperienza da medico alla corte di Ali Pascià Tepelena. Lui si mise al lavoro sotto un contratto triennale alla cui fine riuscì a compiere la traduzione del Vangelo Secondo Matteo. L'opera pubblicata nel 1824 con i caratteri greci rimase lontano dalla maggioranza degli albanesi non essendo a conoscenza di questi caratteri. Comunque l'atto presentava un grande passo nella tortuosa via dell'unificazione della lingua albanese

\section{L'Operato in Sintonia del Rev. A. Thomson e dell'intelettuale K. Kristoforidhi Pose le Basi della Lingua Scritta Albanese}

Subito dopo la Guerra di Crimea, l'attività dei missionari protestanti conobbe un rinvigorimento nella sua attività, e fino al 1878, loro erano i principali artigiani della letteratura in lingua albanese. (Clayer, 2012, p. 189). Furono loro che nel 1858, investirono nella nuova edizione ad Atene della traduzione bilingue del Nuovo Testamento. II rapporto tra i missionari della S.B.B.S. e gli intelettuali albanesi proseguì negli anni a venire tra due personalità Rev. Alexander Thomson e Kostandin Kristoforidhi.

Thomson studiò nell' Università St. Andrew e nel New College di Edinburg. Come missionario della Chiesa Libera di Scozia lui servì presso la comunità ebrea a Costandinopoli. Dopo diciasette anni di servizio col consenso del suo comitato ad Edinburg fu trasferito alla S.B.B.S. Rev. A. Thomson venne nominato direttore della Società per la Turchia la quale comprendeva anche la regione albanese. Nel viaggio di ricognizione che intraprese in Albania, lui si convinse delle oportunità che vari stratti della popolazione albanese accetassero il messaggio evangelico. Quel viaggio comprendeva la maggior parte dei centri abitati della parte occidentale delle terre degli albanesi , una parte delle quali oggi fuori dei confini statali. La prima impressione fu l'apatia della popolazione di religione cristiana che secondo Thomson, stava nel fatto dell'intervento dell clero ortodosso greco. "In Elbasan tra la popolazione generale c'era tanta apatia. Infatti non abbiamo venduto nemmeno una coppia delle Scritture a qualche cristiano, ma tutte le otto copie che abbiamo venduto furono comprate dai mussulmani. (Thomson, 2002, p. 20) II caso riguadava la precedente visita del missionario Riedel nel 1864, il quale aveva venduto ai credenti cristiani le copie delle Scritture, ma quando era ritornato nel 1865 il vescovo gli aveva chiesto di ritirarle consegnando anche la somma di denaro ricavata. Spinto dal senso della pace, Riedel le ubbidì. 
Nel rapporto indirizzato alla Società Biblica nel 1867, Rev. A. Thomson scriveva; " Tra tutte le razze, dentro i confini della Turchia Europea, nessuna è stata cosi terribilmente trascurata quanto gli albanesi”. Era memorabile la sua presentazione del tipo dell'educazione offerto alla popolazione albanese a Scutari, la maggiore città del nord Albania. " II fondamentale difetto delle scuole albanesi è che loro tentano di educare la gioventù albanese attraverso una lingua sconosciuta, trascurando quello che capiscono e parlano meglio, l'Albanese. Questa da tanto nell' occhio a uno straniero, ma la situazione viene spiegata dalla mancanza dei libri in quella lingua". (Thomson, 2002, p. 54)

Il secondo ostacolo pel lavoro missionario fu l' atteggiamento ostruzionista della struttura del potere ottomano. Gli accordi internazionali che proteggevano l' attività della Società Biblica, sottoscritto dallo stesso Sultano, venivano ignorati dalle autorità locali. Thomson annotava che : "Il governo ha deciso di impedire sia noi, sia gli americani, ponendoci ogni tipo di ostacolo nel lavoro missionario e particolarmente nella distribuzione della litteratura biblica in Albania". (Quanroud, 1998, p. 62) Nel aprile 1883 Thomson venne informato che le autorità avevano proclamato il divieto dei libri albanesi in dialetto tosk, pubblicati dalla Casa Biblica. Secondo lui riferendosi anche alla situazione in Albania; " dopo un po di tempo gli ordini del governo non avevano nessun valore." (Quanroud, 1998, p. 63) Non curando di tutte queste difficoltà per tutti gli anni del suo servizio Rev. A. Thomson tenne in cuore la questione albanese alla quale dedicò la vità e i suoi beni con l'opera dell' educazione e dell'evangelisazione.

K. Kristoforidhi(1827-1895) provenne da Elbasan, città ombelico dell' Albania. Lui compì gli studi al liceo Zosimea dopo di che maturò intelletualmente al colleggio protestante di Malta. A Janina fu invitato a dare lezioni di lingua albanese al Dr. Johan Von Hann, il maggiore albanologo del dicianovesimo secolo. In un ipotesi si valuta che grazie alle sue conoscenze con i protestanti, fosse stato Hahn a raccomandare Kristoforidhi a loro. Dopo il cambio di lettere e un incontro a Costandinopoli con Rev. A. Thomson, prese a lavorare sotto contratto presso la Società Biblica con la traduzione delle parti della Bibbia in albanese. Dopo il ritorno in Albania, lui intraprese vari viaggi passando per Berat, Elbasan, Scutari ( $\mathrm{i}$ tre centri dei dialetti albanesi), entrando in stretto contatto con la gente e raccogliendo vocaboli e frasi, arrichendo così il suo vocabolario. Le traduzioni delle intere parti della Bibbia furono fatti nei due dialetti albanesi gheg del Nord e tosk del Sud d'Albania . Nel 1866 a Costandinopoli vide la luce l'edizione del volume intitolato "I Quattro Vangeli del nostro Signore Gesu Cristo"; la sua prima traduzione in dialetto gheg. Durante 1868 lui compì la traduzione in dialetto tosk dei "I Salmi ". Dopo quattro anni alla sua collana si aggiunse "Il Nuovo Testamento" in dialetto tosk. Per le scritture nel dialetto tosk usò i caratteri greci, mentre per quelle in dialetto gheg usò i caratteri latini. Lo stesso Kristoforidhi aveva pensato a un alfabeto standartizzato con caratteri greci adattandolo ai bisogni del dialetto tosk e anche quello gegh. (Lloshi, 1974, p. 129) Spinti dall'idea di distribuire quanto più le Scritture Sacre, furono i missionari protestanti a proporre a Kristoforidhi di usare per le edizioni in dialetto gheg l' alfabetto latino con l'aggiunta dei segni dal sistema universale dell' egittologo tedesco Lepsius. Col passare del tempo Kristoforidhi fece la sua causa, l'uso dei caratteri latini, quando in una lettera indirizzata a Nikolla Naço a Bucarest optò per quella variante. (Nergjoni, 2013, p. 24) Lui pubblicò anche la Bibbia nel dialetto tosk e gheg (1872), una grammatica in dialetto tosk (1882) e un ricco vocabolario albanese-greco che vide la luce nel 1904. La traduzione della Bibbia è in uso ancora oggi durante la messa della chiese Ortodosse in una variante moderna pubblicata nel 2005. (Hellstein, 2008, p. 56) Opera di Kristoforidhi ebbe un impatto straordinario sulla cultura albanese dell'epoca eppure la sua attività da missionario ebbe i suoi limiti. A capire meglio la portata della sua azione ci aiuterebbe l'opinione di due noti albanologhi come Peter Bartl che diceva: " Certamente, lui non era il Luter degli albanesi come a volte è stato chiamato; eppure le sue traduzioni hanno aiutato nella creazione della lingua letteraria albanese". (Bartl, 1995, p. 106) Accanto a questo si aggiunge anche la valutazione dello studioso Robert Elsie: " Queste traduzioni servirono come base di una moderna lingua letteraria in due varianti di dialetti" (Elsie, 2011, p. 342)

\section{La Distribuzione della Letteratura Biblica Innescò il Risveglio della Coscienza Nazionale}

Come affermava Edwin Jacques nella sua opera " Gli Albanesi", la letteratura nella lingua albanese appena esisteva essendo vietata sia dalle autorità di stato ottomane, sia dalla Chiesa Ortodossa. II sistema del "milet" con cui la "Sublime Porta" sanciva i rapporti tra essa e i vari popoli e le varie comunità confessionali, considerava gli albanesi mussulmani ottomani e quelli cristiani come greci, serbi e italiani. Sulla stessa onda anche la chiesa ortodossa optava per l'uso solo del greco nella liturgia opponendosi all'uso della lingua albanese. Per di più non esisteva un alfabetto standartizzato. Eppure la S.B.B.S. ebbe modo di sorpassare gli ostacoli. Le idee della Società Biblica concordarono con la principale caratteristica del nazionalismo albanese che fu quella, di maturarsi basandosi principalmente sulla lingua e non sulla fede come nel caso degli altri nazionalismi della regione. (Clayer, 2012, p. 11). Le edizioni in albanese ebbero una particolare influenza su una ristretta cerchia di albanologhi stranieri e uomini istruiti albanesi i quali si interessavano della lingua albanese. Proprio in quelle edizioni, i lingusti Xylander e Hahn trovarono appoggio pel compimento dei loro studi. (Clayer, 
2012, p. 164) Paradossalmente era il clero mussulmano albanese o l' èlite dirigente albanese convertita all' islam, più propensi verso la parola sacra nei Vangeli. Thomson raccontava che durante il suo viaggio missionario, alla fermata di Kruja, città centrale dell' Albania, il collega di missione Dr. Koelle ebbe un cortese scambio di opinioni con un mullah, autorità del clero mussulmano, alla fine del quale dopo che gli s'era oferta una Bibbia lui rispose: "Come vedete signore, io sono un uomo povero, ma è cosi grande il rispetto nei confronti del profetta Jesu, che io compra questo libro". (Thomson, 2002, p. 20) Un fatto del genere ha toccato anche la diaspora albanese a Costantinopoli. Nel suo rapporto riguardante l'anno 1868, il rappresentante della Società Biblica con sede alla capitale ottomana informava che i Salmi erano tanto richiesti dalla popolazione albanese a Costantinopoli. Lui sottolineava sopra tutto che le Scriture e le grammatiche venivano acquistate anzi anche dai mussulmani del sud "tosk" e del nord "gheg" che a volte erano negli alti ranghi dirigenti dell' impero. Alcuni di loro, funzionari dei tribunali " kadi e myderis" facevano pubblico il loro impegno a distribuire quelli libri senza curarsi delle conseguenze.

L' Elite intelettuale albanese vedeva nei Vangeli in lingua albanese un mezzo di enorme importanza per il risveglio della coscienza nazionale. Nel 1912 una grande personalità come Mid-hat Frashëri affermava che "La Casa Biblica era onorata e lodata da tutti i patrioti albanesi sia cristiani, sia mussulmani (Clark, 2012, p. 7)

\section{II Congresso di Manastir e la Standartizzazione della Lingua Albanese}

Dopo la presa di potere da parte dei Turchi Nuovi e la dichiarazione di Costituzione emerse la possibilità per l'èlite intelettuale albanese di investire nel risolvere il problema piu importante culturale del momento " la Standartizazzione dell' Alfabeto Albanese." Fu la Società "Bashkimi" (Unione) che prese il compito di organizzare il Congresso il 14 novembre 1908 nella sua sede a Manastir. Secondo prof. Bedri Dedja parteciparono intorno 50 delegati inviati dalle società delle colonie albanesi da est fino a ovest. Tra i delegati c'era anche Grigor Cilka e Gjergj Qiriazi. Cilka era presente in qualità di delegato dalla scuola di ragazze di Korça; lui era anche pastore della Chiesa Evangelista di Korça. T. R. Hodgson della Società Biblica seguiva via epistolare il corso dei lavori. Dopo aver letto il rapporto mandato da Gjergj Qiriazi valutò che il risultato rapresentava un lavoro difficile e molto attento all' ortografia, la quale era meravigliosa. Qiriazi fu scelto vicepressidente. Nel suo discorso lui optava per la variante alfabetica di Costandinopoli. Al terzo giorno del congresso fu scelto un comitato di undici persone con pieni poteri di scegliere la variante universale. Gjergj Qiriazi e Grigor Cilka furono tutti e due scelti. II 20 novembre dalla comissione fu presa la decisione di optare per la variante della società " Bashkim" con alcune modifiche. Nel rapporto del 16 gennaio 1909 indirizzato alla "Casa Biblica" Qiriazi sottolineava che

: " pressapoco tutti i delegati che erano presenti al congresso espressero tanta gratitudine verso la Società Biblica per tutto ciò che ha fatto verso la nazione... I servizzi di questa società non saranno mai dimenticati dagli albanesi pieni di gratitudine, perché nei tempi quando ogni libro in lingua albanese era vietato, solo la Società Biblica poteva circolare i libri nella nostra lingua materna. Tra quelli che hanno espresso gratitudine c'erano anche tanti mussulmani, la maggioranza dei quali mi ha chiesto di portare i loro calorosi ringraziamenti per quello che avete fatto e farete nel futuro" (Young , 2011, p. 38)

\section{Gjerasim Qiriazi e la Fratelanza Evangelica}

Nel 1873 due missionari protestanti arrivarono nella città di Manastir. (Hellstein, 2008, p. 57) I due missionari erano in missione per conto del Bordo Americano. La città fu scelta per il suo peso politico come centro di vilajet e per il suo livello culturale relativamente alto. Gjerasim il quale secondo le testimonianze documentarie nacque il 18 ottobre 1858 e morì il 1894, fu uno dei loro primi adepti. II 19 agosto del 1877 dopo aver superato l'esame , Gjerasim fu accettato nella Fratelanza Evangelica di Manastir. Quella via fu seguita più tardi anche da sua fratello Gjergj e dalle due sue sorelle Parashqevi e Sevasti. I missionari protestanti organizzarono classi speciali per gli allievi i quali potevano proseguire dopo gli studi in una delle scuole medie della missione. Gjerasim cominciò gli studi presso il coleggio di Samokov nel 1878 e lo concluse nel 1882. La sua classe era l'ultima che accettò studenti le spese dei quali venivano affrontate dalla missione protestante. Durante il periodo di studio a Samokov un particolare influenza ebbe su di lui John House il quale fu fondatore della scuola agricola americana a Salonico. Nell' anno 1882 Gjerasim partecipò alla riunione della missione protestante del Bordo Americano a Costandinopoli dove fu conferito di prendere cura di una piccola congregazione bullgara a Scopie. Ormai Gjerasim era un " predicatore con la licenza" e aveva il diritto di dare lezioni sulla Bibbia. Era parte della tradizione che gli studenti che avevano usufruito di una piena borsa di studio dal collegio dovevano servire sotto un piccolo stipendio per un certo periodo. Durante il viaggio per Skopie lui si fermò a Manastir ad incontrare i familiari e i compagni di fede. Propio qui avvenne "la rottura" con la missione precedente. Fu il missionario americano John Berd che informò Gjerasim sull' attività del Reverendo Thomson, il quale dopo il permesso concesso dai missionari 
del Bordo Americano, attraverso una lettera del 27 gennaio 1883 chiese la cooperazione di Gjerasim. Nella lettera di risposta indirizzata a Thomson Gjerasim si esprimeva che " era toccato dalla volontà di Thomson di predicare il Vangelo tra gli albanesi" e per conoscere meglio le necessità del paese proponeva " che insieme ad un suo amico di visitare i villaggi e le principali città dell' Albania portando con se anche i libri necessari". (Quanroud, 1998, p. 62) Con un telegramma del 3 maggio 1883 Thomson confermò a Gjerasim il suo contratto come colporter " missionari che vendono le copie della Bibbia" con la Società Biblica. II 4 maggio 1883 Gjerasim parte da Scopie per arrivare dopo dieci giorni a Korça via Manastir. Sull' atteggiamento dei suoi compatrioti verso le religioni, lui si espresse : "Gli albanesi sono nobili e non fanatici come le altre nazioni della Turchia" (Quanroud, 1998, p. 64) Conseguenza di tale atteggiamento fu la vendita di tanti volumi del Nuovo Testamento nel dialetto tosk e gheg, della grammatica in dialetto tosk di Kristoforidhi, del Catechismo per bambini in dialetto tosk. Gjerasim non si fermò solo a questa. Nel rapporto della data del 21 agosto 1884 da Manastir un prete della chiesa uniata si riferiva di un giovane protestante albanese ; "il quale si occupava dello studio della sua lingua materna, alla quale si era dedicato con anima e corpo, il quale stava cercando di preparare l'edizione di una nuova originale grammatica". Compiendo la sua missione da pastore protestante lui predicava in lingua albanese nell' edificio della scuola greca dove veniva seguito da un pubblico molto attento. (Young, Perendim- Lindje; nje dore miku, 2008, p. 81) Per oltre un decennio la sua attività missionaria fece decollare gli ideali protestanti e nazionali diventanto una figura di spicco tra i patrioti albanesi. Nel 1889 per suggerimento del poeta del Rinascimento Albanese Naim Frasheri, cominciò a prepare l'edizione dei primi cinque libri della Bibbia, addatatti come testi scolastici. Nel fratempo lui allacciò contatti e raccolse fondi dalle società delle colonie albanesi e dai patrioti come, i Fratelli Frasheri, Kristo Dako, Nikolla Naço, Mihal Grameno.

L' apice dell' attività missionaria avvenne con la fondazione della Chiesa Evangelica a Korça il 14 novembre 1892. Contemporaneamente lui fondò anche la società "La Fratelanza Evangelica dell' Albania " e pubblicò il primo numero del giornale della società intitolata "Lettere della Fratelanza". Oltre a questo cooperò anche con il dirigente della prima scuola albanese Pandeli Sotiri e un suo maestro Athanas Sina.

Diversamente da tanti uomini istruiti albanesi, Gjerasim tornò in Albania a contribuire in mezzo al suo popolo. Durante i suoi numerosissimi viaggi lui insegnò e predicò in albanese, portando il sentimento della speranza, e faccendo rinascere ai suoi compatrioti il senso rinato dell' identità nazionale.

\section{Le Sorelle Qiriazi e la Scuola Biblica delle Ragazze}

Alla fine del XIX secolo e all'inizio del XX secolo la regione albanese veniva caratterizzata da una società di carattere patriarcale. Lo spazio d' azione delle donne albanesi era abbastanza scarso. La cultura della divisione e differenzazione tra i due generi imposta dalle autorità ottomane comprimeva di più la libertà femminile. In queste condizioni il messaggio protestante portava un vento di cambiamento nell' atteggiamento verso le donne mai visto in quella regione. Sarebbe stato la donna protestante albanese che avrebbe affrontato le sfide poste davanti alla società.

Le sorelle Qiriazi ruppero con il tabu sull' istruzione superiore delle giovani albanesi, andando a studiare nel collegio protestante "Robert" di Costantinopoli. Dopo il ritorno a Korça Sevasti con l'aiuto del fratello Gjerasim e dei missionari protestanti fondarono " La Scuola delle Giovani Albanesi" nel 1891, con Sevasti come direttrice. Dopo la morte di Gjerasim, le due sorelle continuarono a dirigere la scuola delle ragazze. Con la chiusura della scuola per ragazzi nel 1902 per opera delle autorità turche, la scuola diretta dalle sorelle Qiriazi era l' unica instituzione dove si insegnava in lingua albanese. II dissenso di coloro che erano contro la lingua albanese fu cosi duro, che le due sorelle videro come l' unica via di salvezza l' appoggio alla copia missionaria Kennedy, i quali diedero un grande contributo alla difesa della scuola delle giovani albanesi.

Anche se la scuola rimanesse con un' unica allieva, io non lo chiuderò" rispose Sevasti Qiriazi al vescovo ortodosso greco. (Dishnica, 2007, p. 73)

Per colmare i bisogni didattici si mise al lavoro anche l'altro fratello Gjergj Qiriazi, il quale raccolse tutta la composizine letteraria creata da Gjerasim e da lui stesso, e la pubblicò nell' anno 1902 sotto il titolo " Cristomazia". Questa edizione influenzò all' arricchimento della letteratura del rinascimento nazionale albanese. Testimoniando la situazione, Gjergj Qiriazi scriveva; " C'è un grande bisogno dei libri in albanese con materiale scelto come la Cristomazia, in modo che i poveri albanesi ne approfittino, mentre aspettano a bocca aperta ogni nuovo e interessante libro." (Dishnica, 2007, p. 74) Oltre a questo lui fece preparare due altri libri "Cristomazia per i viaggiatori" e "Storia della Schiavitù" , considerandoli utili per la mente di ogni albanese.

Sulla stessa scia Sevastia addattò dei testi scolastici contemporanei, cercando di farli affini alla mentalità degli allievi albanesi. Secondo lo studioso Dhimiter Dishnica, I' attività di Sevasti e delle altre maestre, ebbe il ruolo di un centro metodico e di un instituzione specializzata nel compimento dei testi scolastici. Come annotava la stessa Sevasti 
nei suoi ricordi " $C$ ' è voluto lavorare giorno e notte per colmare la richiesta dei testi scolastici in albanese. Mi sento fiera e felice che con tutte le difficolta siamo riusciti a compilare, fare le bozze e alla fine legare tutti i testi di cui la nostra scuola aveva bisogno". Dai suoi contemporanei veniva tanto valutato il suo diapason culturale, i suoi chiari scopi nazionali, la volontà di accrescere il livello della Scuola delle Giovani Albanesi. "I testi di storia, arimetica e fisica preparati da lei erano migliori di quelli precedenti." Con questi termini venivano valutati nella correspondenza epistolare tra due personalità politiche e intellettuali albanesi Thimi Marko e Pandeli Evangjeli. Per la sua età di soli 27 anni lei rapresentava un caso unico per tutto il periodo del rinascimento nazionale albanese. (Dishnica, 2007, p. 74) II console austro-ungarico Krall, citando u rapporto di Gjergj Qiriazi, qualificava questa scuola come il nido di educazione per le future maestre. (Dishnica, 2007, p. 77) La scuola delle giovani fu il nocciolo della scuola nazionale albanese per la preparazione della nuova generazione delle maestre. Secondo Sevasti Qiriazi; " Una delle intenzioni della scuola era di allenare tutte le allieve in modo che un giorno potessero essere in grado di adempire il mestire del maestro perchè solo in questo modo avremmo potuto distribuire l'educazione anche nelle regioni più remote del nostro paese".

Le due sorelle si impegnarono anche negli avvenimenti più importanti che convolsero la vita politica e intelettuale della nazione albanese. Nel Congresso di Manastir Parashqevi ebbe l' onore di essere l' unica delegata femmina e la prima donna che prese parte in un' assemblea panalbanese. Lei servì da segrettaria e nel suo discorso nel congresso la sua opzione sul alfabeto era di uno unico, accettato da tutti.Nel 1909 Parashqevia pubblicò l' Abbecedario per le Scuole Elementari con l'alfabeto standatizzato del Congresso di Manastir, diventandosi così la prima donna albanese che pubblicava edizioni scolastiche in albanese. Lei compose una canzone sull' alfabeto la quale diventò un inno nazionale. Parashqevia fu la fondatrice della società "Ylli i Mëngjesit" (Stella del Mattino) nel 1909, la quale viene riconosciuta come la prima società delle donne albanesi. Lo scopo era di ampliare la scolarizzazione tra le donne albanesi. L' attività della società fu ramificata nelle altre città dell' Albania, distribuendo giornali e libri nella lingua albanese. (Clark, 2012, p. 25) Sevasti Qiriazi notava che " gli albanesi di Manastir diedero prova di non poter essere denazionalizzati o assimilati dalle altre nazionalità" (Dishnica, 2007, p. 67) In questa missione tutta la famiglia Qiriazi trovò appoggio nella missione protestante.

\section{Gli Evangelisti si Impegnano Politicamente a Favore dell' Indipendenza Albanese}

L'opinione americana fu toccata profondamente dalla malvaggia repressione che subì la popolazione albanese dal sud fino al nord durante le ribellioni del 1910-1911. Alcune persone altruiste fondarono " II fondo per la salvezza dell' Albania"con sede alla città di New York, dove assicurarono i servizi del giornalista William Howard come segretario generale. II suo più stretto collaboratore era il Reverendo Frederick Lynch, cassiere dell' organisazione ed editore del periodico "Christian Work and Evangelist." Quel fondo veniva aiutato finanziaramente da un gruppo di filantroppisti americani tra i quali spiccava Charles Crane. Nell' estate del 1911 lui intraprese un viaggio tra Elbasan, Tirana e Scutari. Riguardo la sua opera di benefattore durante quel viaggio, Sevasti Qiriazi scrisse nella sua Autobiografia non pubblicata che: " che tante persone gli sono grate del cibo e del tetto sopra la testa." I giornali albanesi " Drita" e "Leka" nei loro rapporti notavano che l' aiuto di Crane arrivava fino a mezzo milione di franghi d'oro. Quando nel 1912 la scuola delle ragazze a Korça aveva bisogno di ampiare lo spazio, fu propio Crane che diede un generoso contributo.

La sua opera fu valutata anche dagli albanesi delle colonnie. Nella riunione del 29 giugno 1911 della società "Deshira" ( Desiderio) con sede a Sofie l' esempio del missionario americano nella raccolta dei fondi all' aiuto delle popolazioni albanesi ribellate ai turchi veniva raccomandato anche agli albanesi emigranti. (Asllani, 2009, p. 122)

Anche altri due missionari evangelici Phinneas Kennedy e Charles Erickson presero parte al movimento politico per la diffesa dell' integrità territoriale dell' Albania. Durante la situazione confusa del 1912 era Phineas Kennedy che teneva informato sul corso della situazione politica in Albania Ericson mentre lui faceva l'avvocato degli interessi nazionali albansi presso i politici occidentali.

Durante il conflitto mondiale le sorelle Qiriazi spostarono la loro attività negli Stati Uniti . Parashqevi Qiriazi ebbe la responsabilità dell' edizione mensile del periodico "Yll'i Mëngjesit" (Stella del Mattino), dal 1917 fino al 1920. I temi degli articoli riguardavano la difesa dell' indipendenza e delle tradizioni albanesi. In particolare le edizioni avevano grande riguardo per quanto riguarda la problematica della donna albanese. Allo stesso tempo lei era anche redattore dell' "Albania" giornale del Partito Nazionale Albanese negli Stati Uniti, l' edizione del quale cominciò nel marzo del 1918, in Wochester Massachusets. II Partito nazionale albanese chiese a lei di essere la loro rappresentante per l'Albania nella Conferenza di Pace a Parigi. Lei era l'unica delegata donna e veniva accompagnata da due personalità del mondo albanese il patriota Mihal Grameno e il missionario Charles Erickson. Nel settembre del 1920 lei fa il viaggio di ritorno in Albania per aiutare la croce rossa nel loro programma scolastico.

Questa attività non poteva passare inosservata all' attenzione dei patrioti albanesi. Uno di loro Mid-hat Frasheri si 
espresse che: " Onore e tanta gratitudine alla Società Biblica Britannica e Straniera per i servizi che ha fatto al paese e alla nostra litteratura... Infatti è impossibile , parlare della lingua e litteratura albanese e non ricordarsi delle loro fatiche e le loro sofferenze, davanti a così tante difficoltà" (Clark, 2012, p. 11)

Lo stesso Onorevole Ismail Qemalli, fondatore dello Stato Albanese durante la sua presenza a Londra con lo scopo di difendere l' integrità territoriale del paese davanti alla Conferenza degli Ambassadori di Londra a metà dell' anno 1913 , fece una visita ad alcuni cristiani evangelisti inglesi ( a ringraziargli dell' aiuto che avevano dato alla questione albanese"(Young, 2011, p. 55)

\section{Conclusione}

II movimento evangelico in Albania acquisì con il passare del tempo un carattere sempre di più nazionale. I protestanti albanesi diedero un contributo di primo piano alla creazione delle instituzioni le quali maneggiarono l' attività religiosa. La simbiosi degli interessi comuni degli attivisti protestanti con queli delle altre religioni promosse l' unione nazionale evitando così una ulteriore disgregazione nazionale. L' Albania è l' unico caso nelle regioni balcaniche dove il movimento protestante si incorporò pienamente alla questione nazionale. II movimento contribuì alla questione nazionale facendolo guadagnare il supporto delle potenze occidentali. Questa attività costruttiva della comunità protestante albanese ampliata nel tempo fino ai nostri giorni gli valse il titolo della "quarta comunità religiosa " col decreto di legge del governo albanese del 22 dicembre 2010.

\section{Bibliography}

Asllani, U. (2009). Kristo Luarasi, Jeta dhe Vepra. Tirana: Plejad.

Bartl, P. (1995). Albanien. Regensburg: Friedrich Pustet.

Clark, J. (2012). 1912 e il Vangelo. Tirana: Vernon.

Clayer, N. (2012). Ne Fillimet e Nacionalizmit Shqiptar. Tirane: Përpjekja.

Dishnica, D. (2007). Miqte e Shqiperise. Tirana: CAD.

Elsie, R. (2011). Dizionario della Storia Albanese. In R. Elsie, Dizionario della Storia Albanese (p. 342). Tirana: UEGEN.

Hellstein, D. (2008). Historia e Krishterimit ne Shqiperi. Tirana: Vernon.

James, C. (2012). 1912 dhe Ungjilli. Tirana: Vernon.

Lloshi, X. (1974). Te dhena dokumentare te reja mbi veprimtarinë e K. Kristoforidhit . In Studime Filologjike 2 (p. 129). Tirana: Akademia Shqiptare e Shkencave.

Nergjoni, F. (2013). Kristoforidhi. Tirana: Naimi.

Quanroud, J. (1998). La Vita di Gjerasim Qiriazi. Tirana: Kartë e Pendë.

Thomson, A. (2002). Percorsi Biblici nella Vecchia Albania. Tirana: Vernon.

Young, D. (2008). Perendim- Lindje; nje dore miku. Tirana : Vernon.

Young, D. (2011). Il Movimento Protestante tra gli Albanesi s1908-1991. PrishtinaTenda. 